Elin Roos, BMedSc

Daniela Mariosa, MSc

Caroline Ingre, $\mathrm{MD}, \mathrm{PhD}$

Cecilia Lundholm, MSc

Karin Wirdefeldt, MD,

$\mathrm{PhD}$

Per M. Roos, MD, PhD

Fang Fang, MD, PhD

Correspondence to

D. Mariosa:

Daniela.Mariosa@ki.se

\title{
Depression in amyotrophic lateral sclerosis
}

\section{ABSTRACT}

Objective: To examine the relative risk of depression among patients with amyotrophic lateral sclerosis (ALS), both in terms of depression diagnosis and use of antidepressant drugs, before and after diagnosis.

Methods: We conducted a nested case-control study including 1,752 patients with ALS diagnosed from July 2005 to December 2010 and 8,760 controls based on the Swedish national health and population registers, to assess the associations of depression diagnosis and use of antidepressant drugs with a subsequent risk of ALS. We further followed the patients with ALS after diagnosis to estimate the association of an ALS diagnosis with the subsequent risk of depression and use of antidepressant drugs.

Results: Before diagnosis, patients with ALS were at higher risk of receiving a clinical diagnosis of depression compared to controls (odds ratio [OR] 1.7, 95\% confidence interval [CI] 1.3-2.3), and the highest risk increase was noted during the year before diagnosis (OR 3.5, 95\% $\mathrm{Cl}$ 2.1-5.6). Patients with ALS also had a highly increased risk of depression within the first year after diagnosis (hazard ratio 7.9, 95\% Cl 4.4-14.3). Antidepressant use was more common in patients with ALS than in controls, especially during the year before (OR 5.8,95\% Cl 4.5-7.5) and the year after (hazard ratio 16.1, 95\% Cl 11.5-22.6) diagnosis.

Conclusions: Patients with ALS are at higher risk of depression diagnosis and use of antidepressant drugs both immediately before and after diagnosis. Neurology ${ }^{\circledR}$ 2016;86:2271-2277

\section{GLOSSARY}

ALS = amyotrophic lateral sclerosis; $\mathbf{A T C}=$ Anatomical Therapeutic Chemical; $\mathbf{C l}=$ confidence interval; $\mathbf{F T D}=$ frontotemporal dementia; ICD = International Classification of Diseases; OR = odds ratio.

Approximately $30 \%$ of patients with amyotrophic lateral sclerosis (ALS) show mild cognitive impairment and $15 \%$ present with overt frontotemporal dementia (FTD). ${ }^{1,2}$ The overlap between ALS and FTD presents a continuum of symptoms such as memory impairment, apathy, and behavioral changes, ${ }^{3,4}$ many of which mimic the typical symptoms of depression. ${ }^{5}$

The strong emotions evoked by receiving a serious diagnosis such as ALS may also lead to increased risk of mental illnesses, including depression. Multiple studies have shown that patients with ALS are more likely to develop depression than ALS-free individuals, although the reported prevalence of depression varies greatly. ${ }^{6-13}$ Different measurements of depression together with the varying representativeness of the patient samples used in previous studies may partly explain the different prevalences reported so far.

The aim of the present study was to investigate the occurrence of depression diagnosis and use of antidepressants before and after ALS diagnosis in a nationwide sample of patients with ALS, using a matched random sample of the Swedish population as the reference.

METHODS Study design. Ascertainment of ALS diagnosis. The Swedish Patient Register was established by the Swedish National Board of Health and Welfare in 1964/1965 aiming to collect information on all hospital discharges in Sweden. Since

From the Departments of Medical Epidemiology and Biostatistics (E.R., D.M., C.L., K.W., F.F.) and Clinical Neuroscience (C.I., K.W.), and Institute of Environmental Medicine (P.M.R.), Karolinska Institutet, Stockholm, Sweden; and Department of Neurology (P.M.R.), Division of Clinical Neurophysiology, Oslo University Hospital, Oslo, Norway.

Go to Neurology.org for full disclosures. Funding information and disclosures deemed relevant by the authors, if any, are provided at the end of the article. 
1987, this register has had nationwide coverage for all inpatient care and since 2001 onward also $>80 \%$ of hospital-based outpatient specialist care. Information available in the Patient Register includes the personal identity number, age, sex, dates of hospital admission and discharge or date of outpatient visit, as well as the main and secondary discharge diagnoses.

We defined an ALS case as an individual with at least one inpatient or outpatient hospital visit at which ALS was recorded as a diagnosis in the Patient Register; the first hospital visit for ALS was used as the date of ALS diagnosis. The Swedish revisions of the ICD codes 356,10 (ICD-7 before 1969), 348,00 (ICD-8 1969-1986), 335C (ICD-9 1987-1996), and G12.2 (ICD-10, 1997 onward) were used to identify ALS diagnoses.

Ascertainment of depression diagnosis and antidepressant use. We retrieved the clinical diagnosis of depression from the Swedish Patient Register. Given the fact that information on outpatient hospital visits was only available since 2001 in this register, we ascertained depression diagnosis from January 1, 2001, onward, using the ICD-10 codes F32 (episode of depression), F33 (relapsing depression), and F341 (dysthymia).

We retrieved antidepressant prescription information from the Swedish Prescribed Drug Register, which provides prescription dates, dispensation dates, and total number of dispensations for all pharmaceuticals dispensed in Sweden since July 2005. Drugs are coded according to the Anatomical Therapeutic Chemical (ATC) system. Accordingly, we ascertained antidepressant use from July 1, 2005, onward. Antidepressants examined in the present study included nonselective monoamine reuptake inhibitors (ATC code N06AA; clomipramine, trimipramine, amitriptyline, nortriptyline, maprotiline), selective serotonin reuptake inhibitors (ATC code N06AB; fluoxetine, citalopram, paroxetine, sertraline, fluvoxamine, escitalopram), monoamine oxidase A inhibitors (ATC code N06AG; moclobemide), and other antidepressants (ATC code N06AX; mianserin, mirtazapine, bupropion, venlafaxine, reboxetine, duloxetine, agomelatine).

In the main analysis, we defined users of antidepressant drugs as individuals with $\geq 2$ dispenses of antidepressants, aiming to identify individuals with relatively long-term use of antidepressants. As a result, we defined the first prescription date as the date of antidepressant use.

Depression diagnosis and use of antidepressants were first analyzed separately. "Any depression" was additionally analyzed, defined as either with a depression diagnosis or use of antidepressants.

In a sensitivity analysis, we defined users of antidepressant drugs as individuals with $\geq 1$ dispense of antidepressants.

Study base. Using the individually unique personal identity numbers, we followed all Swedish residents born in Sweden and enlisted in the 1990 Swedish Census or in a previous Census and alive on January 1, $1991(\mathrm{n}=9,154,777)$ individually through cross-linkages to the Swedish Patient, Cause of Death, and Migration Registers. We excluded from the analysis individuals who were diagnosed with ALS, died, or emigrated out of Sweden before July 1, 2005, as well as those with missing information on area of residence in the Census, leaving a study population of 6,337,988 individuals. Each individual contributed person-time to the study base from July 1, 2005, until a new ALS diagnosis, death, emigration out of Sweden, or December 31, 2010, whichever came first.

Nested case-control study: Depression and the subsequent risk of ALS. To study the risk of ALS associated with depression and antidepressant use, we conducted a nested case-control study within the study base. Cases were all individuals diagnosed with ALS between July 2005 and December 2010. We randomly selected 5 controls per case from the study base using incidence density sampling and individually matched the controls to the case on year of birth, sex, and region of residence. We defined index date as the date of diagnosis for cases and the date of selection for controls. In total, 1,752 cases and 8,760 controls were enrolled in the nested case-control study. Information on depression diagnosis and antidepressant use before the index date was ascertained for both the cases and controls. To ensure the ascertainment of antidepressant use for at least 1 year before the index date, we included only individuals with the index date between July 1, 2006, and December 31, 2010, in the analyses of antidepressant use and any depression.

Matched cohort study: ALS and the subsequent risk of depression. We further performed a matched cohort study to compare the risk of depression diagnosis or antidepressant use between patients with ALS and individuals without ALS. We followed both the cases and controls enrolled in the nested case-control study from the index date to the first diagnosis of depression, first prescription of antidepressants, death, or December 31, 2010, whichever came first. We excluded individuals with a diagnosis of depression $(n=307)$ or antidepressant use $(\mathrm{n}=1,403)$ before the index date in the analysis for depression and antidepressant use, respectively. Follow-up of the controls was also censored if they developed ALS during follow-up $(\mathrm{n}=1)$.

Covariates. We obtained information on the highest achieved education at the index date from the Swedish Education Register, categorized as $<9$ years, 9-12 years, or university or doctoral studies. We defined socioeconomic status of the study participants according to the occupation reported in the 1990 Census, categorized as blue collar, white collar, self-employed or farmer, or unclassified.

Statistical analyses. In the nested case-control study, we used the conditional logistic regression model to estimate the odds ratios (ORs) with 95\% confidence intervals (CIs) for the association of depression and the subsequent risk of ALS. To test whether the association differed by time before ALS diagnosis, we performed separate analyses for different time windows ( $>3$ years, third year, second year, and first year) before the index date.

In the matched cohort study, we used the conditional Cox regression model to estimate the hazard ratios with 95\% CIs for the association of ALS with the subsequent risk of depression. Similarly, we separately analyzed the first year and second year after diagnosis to assess whether the association varied over time since ALS diagnosis.

Given the matched study design, all analyses were inherently adjusted for the matching variables. In the full models, education and socioeconomic status were further adjusted. Individuals with missing information on education were excluded from the adjusted analysis.

Analyses were conducted using SAS, version 9.4 (SAS Institute, Cary, NC).

Standard protocol approvals, registrations, and patient consents. The study was approved by the regional ethics review board at the Karolinska Institutet, Stockholm, Sweden.

RESULTS The distributions of the matching variables, education, and socioeconomic status in ALS cases and their matched controls are presented in table 1 . 


\begin{tabular}{|c|c|c|c|}
\hline \multirow[t]{2}{*}{ Table 1} & \multicolumn{3}{|c|}{$\begin{array}{l}\text { Basic characteristics of the patients with ALS and their matched controls: A nested case-control } \\
\text { study in Sweden, July } 2005 \text { to December } 2010\end{array}$} \\
\hline & & ALS cases & Controls \\
\hline Total & & $1,752(100)$ & $8,760(100)$ \\
\hline \multicolumn{4}{|l|}{ Sex } \\
\hline Men & & $992(56.6)$ & $4,960(56.6)$ \\
\hline Women & & $760(43.4)$ & $3,800(43.4)$ \\
\hline \multicolumn{4}{|c|}{ Age at the index date, $y$} \\
\hline$\leq 55$ & & 231 (13.2) & $1,152(13.2)$ \\
\hline $56-65$ & & $454(25.9)$ & $2,277(26.0)$ \\
\hline $66-75$ & & 541 (30.9) & 2,694 (30.8) \\
\hline$>75$ & & $526(30.0)$ & $2,637(30.1)$ \\
\hline \multicolumn{4}{|c|}{ Highest achieved education } \\
\hline$<9 y$ & & 650 (37.3) & $3,454(39.7)$ \\
\hline $9-12$ y & & $722(41.5)$ & 3,371 (38.8) \\
\hline Universi & or doctoral studies & $370(21.2)$ & $1,868(21.5)$ \\
\hline Unknowr & & 10 & 67 \\
\hline \multicolumn{4}{|c|}{ Socioeconomic status } \\
\hline Blue coll & & 671 (38.3) & $3,387(38.7)$ \\
\hline White co & & $750(42.8)$ & $3,546(40.5)$ \\
\hline Self-emp & yed/farmer & $187(10.7)$ & 971 (11.1) \\
\hline Unclassi & & 144 (8.2) & $856(9.8)$ \\
\hline
\end{tabular}

Abbreviation: ALS = amyotrophic lateral sclerosis. Data represent $\mathrm{n}(\%)$.

Before the index date, 77 patients with ALS (4.4\%) were diagnosed with depression, compared to 230 controls (2.6\%) (table 2). After the index date, 38 patients with ALS (2.3\%) were diagnosed with depression, compared to 79 controls $(0.9 \%)$. For cases and controls with the index date during July 2006-2010 ( $\mathrm{n}=8,838), 355$ patients with ALS (24.1\%) used antidepressants compared to 1,048 controls (14.2\%) before the index date. After the index date, 217 patients with ALS (19.4\%) and 190 controls (3.0\%) used antidepressants.

Depression and the subsequent risk of ALS. Depression, defined either as a clinical diagnosis or by antidepressant use, was associated with a higher risk of ALS. Similar associations were noted for men and women but the association was stronger for older individuals (aged 65 years and older) compared to younger individuals; educational level and socioeconomic status did not appear to modify the associations greatly (table 3 ). Within 1 year after depression diagnosis, there was a 3.6-fold risk of ALS. Higher risk of ALS was also noted for the second and third years after depression diagnosis (table 3 ). The same pattern was seen for antidepressant use and "any depression" (table 3). Further adjustments for education and socioeconomic status provided very similar results (table 3).

ALS and the subsequent risk of depression. After the index date, patients with ALS had a higher risk of depression diagnosis as well as antidepressant use than controls (table 4). The risk increase appeared to be greater among individuals at age 65 and older, whereas sex, educational level, and socioeconomic status did not seem to modify the associations greatly (table 4). The increased risks were most pronounced during the first year after diagnosis (table 4). Highly increased risk of antidepressant use was still observed during the second year after ALS diagnosis, whereas the risk increase of depression diagnosis during the second year was not statistically significant (table 4). Patients with ALS had a 16.5-times increased risk of "any depression" during the first year after diagnosis. Analyses adjusted for education and socioeconomic status provided similar results (table 4).

Sensitivity analysis. Using $\geq 1$ dispense of antidepressants as the definition for antidepressant use, 403 patients with ALS (27.4\%) and 1,181 controls (16.0\%) used antidepressants before the index date, 


\begin{tabular}{|c|c|c|c|c|c|c|c|c|c|}
\hline \multirow[t]{3}{*}{ Table 2} & \multicolumn{9}{|c|}{ Depression diagnosis and antidepressant use before and after the index date among patients with ALS and their matched controls } \\
\hline & \multicolumn{3}{|c|}{ Depression diagnosis } & \multicolumn{3}{|l|}{ Antidepressants } & \multicolumn{3}{|l|}{ Any depression } \\
\hline & ALS cases & Controls & $p^{a}$ & ALS cases & Controls & $p^{a}$ & ALS cases & Controls & $p^{a}$ \\
\hline \multicolumn{10}{|c|}{ Years before index date } \\
\hline$\geq 4$ & $22 / 1,752$ (1.3) & $124 / 8,760(1.4)$ & 0.74 & $113 / 1,473(7.7)$ & $483 / 7,365$ (6.6) & 0.16 & $120 / 1,473(8.1)$ & $543 / 7,365(7.4)$ & 0.33 \\
\hline 3 & $13 / 1,730(0.8)$ & 28/8,636 (0.3) & 0.02 & $46 / 1,360$ (3.4) & 219/6,882 (3.2) & 0.71 & 49/1,424 (3.4) & $210 / 7,155$ (2.9) & 0.31 \\
\hline 2 & $13 / 1,717(0.8)$ & $37 / 8,608(0.4)$ & 0.09 & $71 / 1,314(5.4)$ & 220/6,663 (3.3) & $<0.01$ & $68 / 1,356(5.0)$ & $212 / 6,943$ (3.1) & $<0.01$ \\
\hline 1 & $29 / 1,704$ (1.7) & $41 / 8,571(0.5)$ & $<0.01$ & $125 / 1,243(10.1)$ & $126 / 6,443(2.0)$ & $<0.01$ & $127 / 1,229(10.3)$ & $123 / 6,820$ (1.8) & $<0.01$ \\
\hline \multicolumn{10}{|c|}{ Years after index date } \\
\hline 1 & $34 / 1,675$ (2.0) & 28/8,530 (0.3) & $<0.01$ & $176 / 1,118(15.7)$ & $93 / 6,317$ (1.5) & $<0.01$ & $184 / 1,109(16.6)$ & $93 / 6,277$ (1.5) & $<0.01$ \\
\hline 2 & $3 / 775(0.4)$ & $28 / 6,569(0.4)$ & 1.00 & $30 / 407(7.4)$ & $50 / 4,616(1.1)$ & $<0.01$ & 29/404 (7.2) & $54 / 4,588$ (1.2) & $<0.01$ \\
\hline$\geq 3$ & $1 / 377(0.3)$ & $23 / 4,762(0.5)$ & 1.00 & 11/192 (5.7) & $47 / 3,056(1.5)$ & $<0.01$ & 12/192 (6.2) & 49/3,040 (1.6) & $<0.01$ \\
\hline
\end{tabular}

Abbreviation: ALS = amyotrophic lateral sclerosis.

Data represent $\mathrm{n}(\%)$.

${ }^{a}$ The $p$ values are from Fisher test.

whereas 314 patients with ALS (29.3\%) and 280 controls (4.5\%) used antidepressants after the index date. In these analyses, antidepressant use was still associated with a higher subsequent risk of ALS (OR 2.0, 95\% CI 1.8-2.3) and patients with ALS still had a higher risk of antidepressant use (OR 13.7, 95\% CI 10.8-17.4) after the index date compared to controls.

DISCUSSION In this large population-based study, we found that patients with ALS were at higher risk of depression diagnosis and use of antidepressant drugs both immediately before and after ALS diagnosis.

There are several potential explanations for the association of previous depression with an increased risk of ALS. For example, depression may be a prodromal symptom of ALS. Although motor symptoms are traditionally perceived as the first and main symptoms of ALS, ${ }^{14}$ the involvement of nonmotor symptoms both before and after the debut of motor symptoms is likely. Depression may also be an early manifestation of frontal lobe degeneration and may stand for an upper motor neuron sign heralding a more widespread cortical affection. Furthermore, depressive symptoms may also overlap with the symptomatology of cognitive impairment, leading to a potential misclassification of these 2 diagnoses among patients with ALS who have concurrent cognitive impairment or FTD. Besides FTD, the comorbidity of ALS with other neurodegenerative disorders, such as Parkinson disease, may also contribute to the association between previous depression and increased risk of ALS. ${ }^{15}$ Finally, little research has been done regarding the potential psychological stress experienced by patients with ALS between the first symptom onset and the final diagnosis, an interval of 1 year on average. ${ }^{16}$ Depression may therefore also be a result of the psychological distress experienced during the progressive symptom development and the often stressful diagnostic workup before ALS diagnosis. ${ }^{17}$

The highly increased risk of depression during the first year after ALS diagnosis may reflect a severe psychological reaction toward the serious diagnosis. Both increased risk of depression and increased risk of antidepressant use have been reported in patients receiving diagnoses of other life-threatening diseases including breast cancer, although the risk increases were smaller in breast cancer (relative risk 1.7 for depression and 3.1 for antidepressant use). ${ }^{18}$

Previous studies rarely examined the temporal pattern of depression prevalence and were mostly based on small numbers of patients (range 27131). ${ }^{6-13,19-21}$ A recent meta-analysis identified a total of 3,190 patients with ALS from 103 studies on ALS and depression, ${ }^{22}$ whereas in the present study, we were able to enroll 1,752 patients in a single study.

Similarly, previous studies also reported largely varying prevalence of antidepressant use (up to 38\%) among patients with ALS. ${ }^{23,24}$ Patients with ALS may be prescribed with antidepressants for reasons other than depression, such as excessive salivation where the anticholinergic side effect of antidepressants is desirable and emotional outbursts where the patients suddenly and without control cry or laugh. These possibilities likely explain the more pronounced risk increase for antidepressant use after ALS diagnosis, compared to depression diagnosis, as observed in the present study. Use of antidepressants before ALS diagnosis has been less investigated, although an increased use of antidepressants was noted in 2 smaller studies. ${ }^{23,25}$ 


\begin{tabular}{|c|c|c|c|c|c|c|}
\hline \multirow[t]{3}{*}{ Table 3} & $\begin{array}{l}\text { lepression dia } \\
\text { trol study in } S\end{array}$ & $\begin{array}{l}\text { gnosis and ant } \\
\text { weden, July } 2 C\end{array}$ & $\begin{array}{l}\text { tidepressant } \\
005 \text { to } 2010\end{array}$ & Ise with the su & ubsequent risk & of ALS: A \\
\hline & \multicolumn{2}{|c|}{ Depression diagnosis } & \multicolumn{2}{|c|}{ Antidepressant use } & \multicolumn{2}{|l|}{ Any depression } \\
\hline & OR $(95 \% \mathrm{CI})^{a}$ & OR $(95 \% \mathrm{Cl})^{\mathrm{b}}$ & OR $(95 \% \mathrm{CI})^{\mathrm{a}}$ & OR $(95 \% \mathrm{CI})^{\mathrm{b}}$ & OR $(95 \% \mathrm{CI})^{\mathrm{a}}$ & OR $(95 \% \mathrm{CI})^{\mathrm{b}}$ \\
\hline Overall & $1.7(1.3-2.2)$ & $1.7(1.3-2.3)$ & $1.9(1.7-2.2)$ & $2.0(1.7-2.3)$ & $1.9(1.7-2.2)$ & $2.0(1.7-2.2)$ \\
\hline \multicolumn{7}{|l|}{ Sex } \\
\hline Men & $1.6(1.1-2.3)$ & $1.6(1.1-2.4)$ & $2.2(1.8-2.7)$ & $2.2(1.8-2.7)$ & $2.1(1.7-2.6)$ & $2.2(1.8-2.7)$ \\
\hline Women & $1.8(1.3-2.6)$ & $1.8(1.3-2.6)$ & $1.7(1.4-2.1)$ & $1.8(1.5-2.1)$ & $1.7(1.4-2.1)$ & $1.8(1.5-2.2)$ \\
\hline \multicolumn{7}{|l|}{ Age at index date } \\
\hline$\leq 65 y$ & $1.5(1.1-2.1)$ & $1.5(1.1-2.1)$ & $1.7(1.5-2.0)$ & $1.8(1.5-2.1)$ & $1.7(1.4-2.0)$ & $1.7(1.5-2.1)$ \\
\hline$>65 y$ & $2.4(1.5-3.9)$ & $2.4(1.5-3.9)$ & $2.4(1.9-3.1)$ & $2.4(1.9-3.2)$ & $2.4(1.9-3.1)$ & $2.4(1.9-3.1)$ \\
\hline \multicolumn{7}{|l|}{ Highest achieved education } \\
\hline Primary school ( $\leq 9$ y) & $2.2(1.2-3.9)$ & $2.2(1.2-3.9)$ & $1.8(1.4-2.3)$ & $1.8(1.4-2.3)$ & $1.8(1.4-2.3)$ & $1.8(1.4-2.3)$ \\
\hline High school or more ( $>9 \mathrm{y}$ ) & $1.8(1.3-2.7)$ & $1.9(1.3-2.7)$ & $2.1(1.7-2.5)$ & $2.1(1.7-2.5)$ & $2.1(1.7-2.5)$ & $2.1(1.7-2.6)$ \\
\hline \multicolumn{7}{|l|}{ Socioeconomic status } \\
\hline Low & $2.1(1.3-3.7)$ & $2.1(1.2-3.5)$ & $2.2(1.7-2.8)$ & $2.2(1.7-2.9)$ & $2.2(1.7-2.8)$ & $2.2(1.7-2.9)$ \\
\hline High & $1.8(1.1-2.7)$ & $1.7(1.1-2.7)$ & $1.9(1.5-2.3)$ & $1.9(1.6-2.3)$ & $1.9(1.5-2.2)$ & $1.9(1.6-2.3)$ \\
\hline \multicolumn{7}{|l|}{ Years before index date } \\
\hline$<1$ & $3.6(2.2-5.8)$ & $3.5(2.1-5.6)$ & $5.6(4.3-7.3)$ & $5.8(4.5-7.5)$ & $5.9(4.5-7.6)$ & $6.1(4.7-7.9)$ \\
\hline $1-2$ & $1.8(0.9-3.3)$ & $1.8(1.0-3.5)$ & $1.8(1.4-2.4)$ & $1.9(1.4-2.5)$ & $1.8(1.4-2.4)$ & $1.9(1.4-2.5)$ \\
\hline $2-3$ & $2.4(1.2-4.8)$ & $2.5(1.3-4.9)$ & $1.2(0.8-1.6)$ & $1.2(0.8-1.6)$ & $1.3(1.0-1.8)$ & $1.3(0.9-1.8)$ \\
\hline$>3$ & $0.9(0.6-1.4)$ & $0.9(0.6-1.5)$ & $1.3(1.1-1.6)$ & $1.4(1.1-1.7)$ & $1.2(1.0-1.5)$ & $1.3(1.0-1.6)$ \\
\hline
\end{tabular}

Abbreviations: $\mathrm{ALS}=$ amyotrophic lateral sclerosis; $\mathrm{Cl}=$ confidence interval; $\mathrm{OR}=$ odds ratio.

${ }^{a}$ ORs and $95 \% \mathrm{Cls}$ estimated by conditional logistic regression and adjusted for age, sex, and region of residence (matching factors).

${ }^{\mathrm{b}}$ ORs further adjusted for education and socioeconomic status.

The main strength of the present study is the national study sample, complete follow-up, and prospectively and independently collected information on depression diagnosis, antidepressant use, and ALS, eliminating major sources of information and selection biases. However, the definition of depression diagnosis was based on hospital records through inpatient or outpatient specialist visits, leading to a lower prevalence of depression diagnosis compared to antidepressant use, among both patients with ALS and controls. A related potential limitation is the use of ICD-10 codes in ascertaining depression. Although the diagnosis of depression has been reported of good quality in the Swedish Patient Register, ${ }^{26}$ whether or not the underlying diagnosis criteria utilized herein are comparable to studies from other settings is unknown. Furthermore, there was limited information on the clinical characteristics of the patients with ALS and as a result we were unable to examine whether the studied associations varied by clinical characteristics.

Common denominators for ALS and depression, including genetic predisposition, personality traits, environmental exposures, as well as socioeconomic vulnerability, were not measured and analyzed. It might be of special interest to study whether development of depression before ALS diagnosis concurs with FTD or upper motor neuron onset. Since C9orf72 has been known to be associated with both ALS and FTD,${ }^{3}$ it would be beneficial to examine the C9orf72 repeats among patients with ALS who have prediagnostic depression. Proposed ALS risk factors such as lead $(\mathrm{Pb})$, smoking, viral encephalopathy, and head trauma may also contribute to depression..$^{27,28}$ For example, chronic low-dose $\mathrm{Pb}$ intoxication may present symptoms indistinguishable from depression, and elevated $\mathrm{Pb}$ exposure may be linked to symptoms of depression. ${ }^{29,30}$

Patients with ALS were at higher risk of depression than individuals without ALS during the year before and the year after ALS diagnosis. To what extent depression should be considered a prodromal symptom of ALS remains to be elucidated. Whether or not these findings are specific to ALS, relative to other neurodegenerative diseases or fatal conditions, warrants further investigation. 
Table 4 Association of amyotrophic lateral sclerosis with the subsequent risk of depression diagnosis and antidepressant use: A matched cohort study in Sweden, July 2005 to December 2010

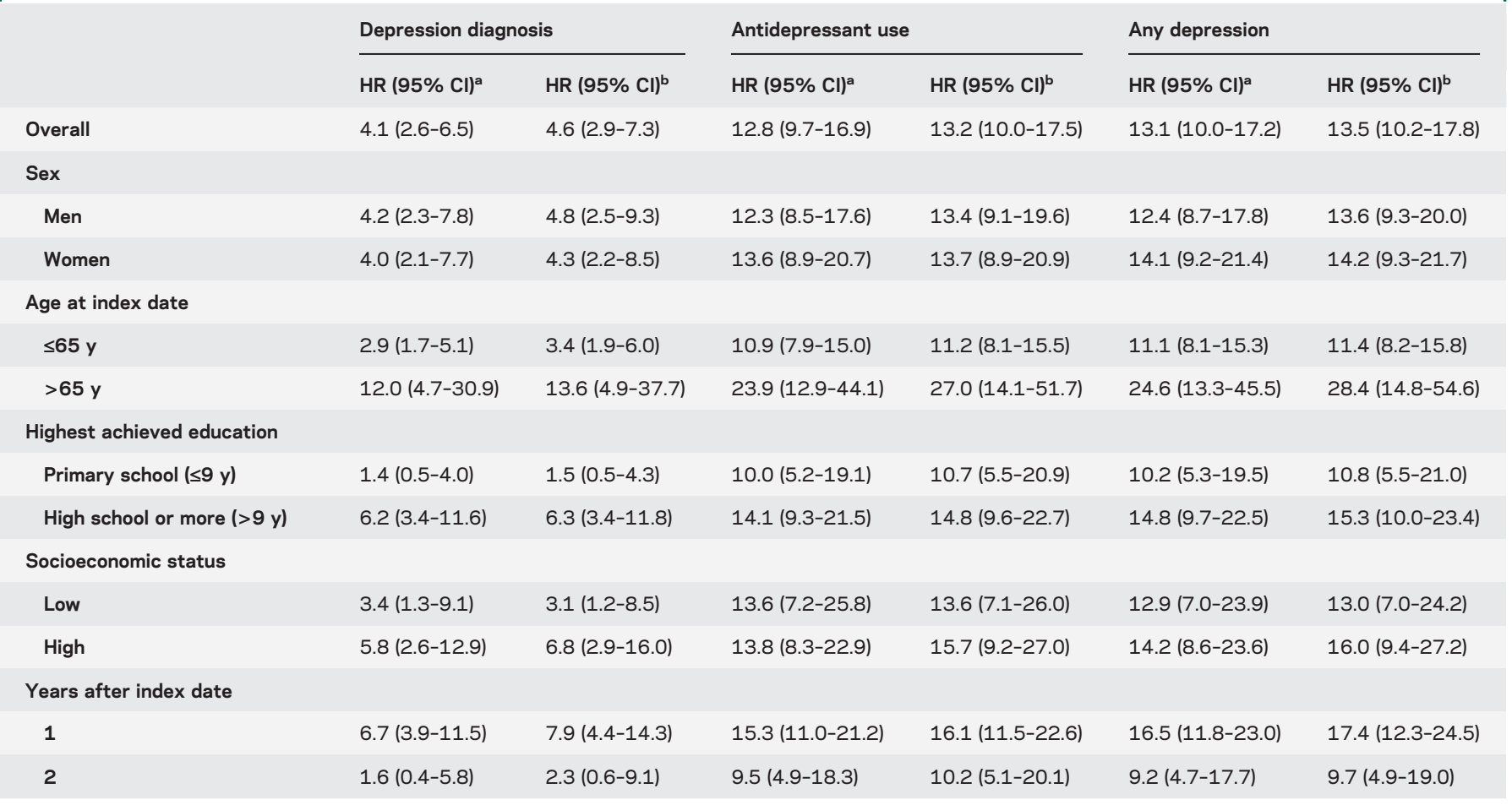

Abbreviations: $\mathrm{Cl}=$ confidence interval; $\mathrm{HR}=$ hazard ratio.

${ }^{a} \mathrm{HRs}$ and $95 \% \mathrm{Cls}$ estimated by Cox regression and adjusted for age, sex, and region of residence (matching factors).

${ }^{\mathrm{b}} \mathrm{HRs}$ additionally adjusted for education and socioeconomic status.

\section{AUTHOR CONTRIBUTIONS}

Study concept and design: E. Roos, Mariosa, Ingre, Lundholm, Wirdefeldt, P. Roos, Fang. Analysis and interpretation of data: E. Roos, Mariosa, Ingre, Lundholm, Wirdefeldt, P. Roos, Fang. Drafting of the manuscript: E. Roos, P. Roos, Fang. Critical revision of the manuscript for important intellectual content: all authors.

\section{STUDY FUNDING}

This study was funded by the Swedish Research Council, the Swedish Society for Medical Research, and the Karolinska Institutet.

\section{DISCLOSURE}

The authors report no disclosures relevant to the manuscript. Go to Neurology.org for full disclosures.

Received October 29, 2015. Accepted in final form March 12, 2016.

\section{REFERENCES}

1. Ringholz GM, Appel SH, Bradshaw M, Cooke NA, Mosnik DM, Schulz PE. Prevalence and patterns of cognitive impairment in sporadic ALS. Neurology 2005;65: 586-590.

2. Ng AS, Rademakers R, Miller BL. Frontotemporal dementia: a bridge between dementia and neuromuscular disease. Ann NY Acad Sci 2015;1338:71-93.

3. Renton AE, Majounie E, Waite A, et al. A hexanucleotide repeat expansion in C9ORF72 is the cause of chromosome 9p21-linked ALS-FTD. Neuron 2011;72:257-268.

4. Lillo P, Savage S, Mioshi E, Kiernan MC, Hodges JR. Amyotrophic lateral sclerosis and frontotemporal dementia: a behavioural and cognitive continuum. Amyotroph Lateral Scler 2012;13:102-109.
5. American Psychiatric Association. Depressive disorders. In: Diagnostic and Statistical Manual of Mental Disorders: DSM-V Washington, DC: American Psychiatric Association; 2013:155-188.

6. Hogg KE, Goldstein LH, Leigh PN. The psychological impact of motor neurone disease. Psychol Med 1994;24: 625-632.

7. Lou JS, Reeves A, Benice T, Sexton G. Fatigue and depression are associated with poor quality of life in ALS. Neurology 2003;60:122-123.

8. Kurt A, Nijboer F, Matuz T, Kubler A. Depression and anxiety in individuals with amyotrophic lateral sclerosis: epidemiology and management. CNS Drugs 2007;21: 279-291.

9. Bungener C, Piquard A, Pradat PF, Salachas F, Meininger V, Lacomblez L. Psychopathology in amyotrophic lateral sclerosis: a preliminary study with 27 ALS patients. Amyotroph Lateral Scler Other Motor Neuron Disord 2005;6:221-225.

10. Olsson Ozanne AG, Strang S, Persson LI. Quality of life, anxiety and depression in ALS patients and their next of kin. J Clin Nurs 2011;20:283-291.

11. Averill AJ, Kasarskis EJ, Segerstrom SC. Psychological health in patients with amyotrophic lateral sclerosis. Amyotroph Lateral Scler 2007;8:243-254.

12. Kubler A, Winter S, Ludolph AC, Hautzinger M, Birbaumer N. Severity of depressive symptoms and quality of life in patients with amyotrophic lateral sclerosis. Neurorehabil Neural Repair 2005;19:182-193.

13. Lule D, Ehlich B, Lang D, et al. Quality of life in fatal disease: the flawed judgement of the social environment. J Neurol 2013;260:2836-2843. 
14. Kiernan MC, Vucic S, Cheah BC, et al. Amyotrophic lateral sclerosis. Lancet 2011;377:942-955.

15. Korner S, Kollewe K, Ilsemann J, et al. Prevalence and prognostic impact of comorbidities in amyotrophic lateral sclerosis. Eur J Neurol 2013;20:647-654.

16. Khishchenko N, Allen KD, Coffman CJ, et al. Time to diagnosis in the National Registry of Veterans with Amyotrophic Lateral Sclerosis. Amyotroph Lateral Scler 2010; 11:125-132.

17. Nzwalo H, de Abreu D, Swash M, Pinto S, de Carvalho M. Delayed diagnosis in ALS: the problem continues. J Neurol Sci 2014;343:173-175.

18. Suppli NP, Johansen C, Christensen J, Kessing LV, Kroman N, Dalton SO. Increased risk for depression after breast cancer: a nationwide population-based cohort study of associated factors in Denmark, 1998-2011. J Clin Oncol 2014;32:3831-3839.

19. Chen D, Guo X, Zheng Z, et al. Depression and anxiety in amyotrophic lateral sclerosis: correlations between the distress of patients and caregivers. Muscle Nerve 2015;51: 353-357.

20. Korner S, Kollewe K, Abdulla S, Zapf A, Dengler R, Petri S. Interaction of physical function, quality of life and depression in amyotrophic lateral sclerosis: characterization of a large patient cohort. BMC Neurol 2015; 15:84.

21. Jelsone-Swain L, Persad C, Votruba KL, et al. The relationship between depressive symptoms, disease state, and cognition in amyotrophic lateral sclerosis. Front Psychol 2012;3:542.
22. Pagnini F, Manzoni GM, Tagliaferri A, Gibbons CJ. Depression and disease progression in amyotrophic lateral sclerosis: a comprehensive meta-regression analysis. J Health Psychol 2015;20:1107-1128.

23. Pisa FE, Logroscino G, Casetta A, et al. The use of antidepressant medication before and after the diagnosis of amyotrophic lateral sclerosis: a population-based cohort study. Neuroepidemiology 2015;44:91-98.

24. Atassi N, Cook A, Pineda CM, Yerramilli-Rao P, Pulley D, Cudkowicz M. Depression in amyotrophic lateral sclerosis. Amyotroph Lateral Scler 2011;12:109-112.

25. Rabkin JG, Albert SM, Del Bene ML, et al. Prevalence of depressive disorders and change over time in late-stage ALS. Neurology 2005;65:62-67.

26. Fazel S, Wolf A, Chang Z, Larsson H, Goodwin GM, Lichtenstein P. Depression and violence: a Swedish population study. Lancet Psychiatry 2015;2:224-232.

27. Ingre C, Roos PM, Piehl F, Kamel F, Fang F. Risk factors for amyotrophic lateral sclerosis. Clin Epidemiol 2015;7:181-193.

28. Bombardier CH, Fann JR, Temkin NR, Esselman PC, Barber J, Dikmen SS. Rates of major depressive disorder and clinical outcomes following traumatic brain injury. JAMA 2010;303:1938-1945.

29. Mason LH, Harp JP, Han DY. Pb neurotoxicity: neuropsychological effects of lead toxicity. Biomed Res Int 2014;2014:840547.

30. Rhodes D, Spiro A III, Aro A, Hu H. Relationship of bone and blood lead levels to psychiatric symptoms: the normative aging study. J Occup Environ Med 2003;45: $1144-1151$.

\section{New! AAN Transforming Leaders Program}

Are you an established AAN member neurologist $10+$ years out of residency who wants to move your career to the next level? Do you want to become a future leader at the AAN and in the field of neurology? Apply for the new, elite AAN Transforming Leaders Program by visiting AAN.com/view/TransformingLeaders. Application deadline is July 1, 2016.

\section{Save These Dates for AAN CME Opportunities!}

Mark these dates on your calendar for exciting continuing education conferences by the American Academy of Neurology.Learn more at AAN.com/conferences.

\section{Sports Concussion Conference}

- July 8-10, 2016, Chicago, IL, at the Hilton Chicago

\section{Fall Conference}

- October 14-16, 2016, Las Vegas, NV, at the Cosmopolitan of Las Vegas 